\title{
CREATIVIDAD CIENTÍFICA Y ALTA HABILIDAD: DIFERENCIAS DE GÉNERO Y NIVEL EDUCATIVO
}

\section{Scientific Creativity and High Ability: Gender and academic level differences}

Fernando Javier Esparza Molina, María José Ruiz Melero, Mercedes Ferrando Prieto, Marta Sainz Gómez y María Dolores Prieto Sánchez

Universidad de Murcia

Correo-e: fjavier.esparza@um.es; mariajose.ruiz4@um.es; mferran@um.es; m.gomez@um.es; lola@um.es

Recepción: 5 de noviembre de 2014

Envío a informantes: Io de noviembre de 2014

Aceptación definitiva: ro de marzo de 2015

Biblid. [02I4-3402 (2015) (II época) n. ${ }^{\circ}$ 2I; 49-62]

Resumen: El objetivo del trabajo es estudiar las diferencias de género y nivel educativo en creatividad científica en una muestra de estudiantes de altas habilidades (superdotados y talentos). En el estudio han participado 78 alumnos de Educación Secundaria Obligatoria (edades comprendidas entre I2 y i6 años). El instrumento utilizado para evaluar la creatividad científica ha sido el Test de Habilidad de la Creatividad Científica (Sak y Ayas, 20II) dirigido a alumnos de entre in y i4 años. El marco teórico de este instrumento contempla tres dimensiones: las habilidades de creatividad general (fluidez, flexibilidad y compuesto creativo); las habilidades científicas (generación de hipótesis, diseño de experimentos y evaluación de la evidencia); y el conocimiento de los alumnos en las áreas de ciencias. La prueba goza de unas adecuadas características psicométricas, siendo el índice de fiabilidad alfa de Cronbach igual a .848 (Sak y Ayas, 20I3). Respecto a las diferencias de género, los resultados muestran que los chicos obtuvieron puntuaciones significativamente más altas que las chicas en la tarea de Gráfico de interacción, que evalúa la generación de hipótesis de forma interdisciplinar en ciencias. En cuanto al nivel educativo, los datos indican que los alumnos de los niveles educativos superiores obtienen puntuaciones significativamente más elevadas en la tarea de Cadena Alimentaria, que valora la evaluación de la evidencia en el área de ecología.

Palabras Clave: alta habilidad; superdotación; talento; género; curso.

AвsтRACт: The purpose of this study was to investigate the influence of gender and educational level on scientific creativity among gifted/talented students. A cohort of 
78 secondary school students from 12 to 16 years old participated in this research. The scientific creativity was measured using the Creative Scientific Ability Test (Sak \& Ayas, 2oir) designed for secondary school students from in to I4 years old. Its theoretical framework sets up the measurement of a three dimensional structure: general creative abilities (fluency, flexibility and creativity), scientific creative abilities (hypothesis generation, hypothesis testing and evidence evaluation) and scientific knowledge. This test has the right adequate psychometric properties with a Cronbach's alpha coefficient of 0.848 (Sak \& Ayas, 20I3). Results indicated that male students scored significantly higher in a task named Interaction Graph which measures hypothesis generation in interdisciplinary science. The analysis also showed that students involved in upper education levels scores significantly higher in general fluency and in the task called The Food Chain which measures evidence evaluation in the area of ecology.

KEY wORD: High ability; gifted; talented; gender; education level.

\section{Introducción}

Marco teórico

$\mathrm{V}$

IVIMOS EN UNA SOCIEDAD TECNOLÓGICA EN LA QUE EL CAPITAL HUMANO juega un papel fundamental en el desarrollo de dicha sociedad. Como afirma Duschl (1997) la mayoría de los estudiantes de ciencias (e incluso los profesores) manejan abundantes datos e informaciones sobre las ciencias. Sin embargo, los alumnos siguen repitiendo y memorizando hechos y datos, sin generar conocimientos o realizar las transferencias oportunas de dichos conocimientos, lo cual supone una deficiencia en el proceso de enseñanza-aprendizaje de las ciencias (Duschl, I997). Según la definición de los especialistas convocados por la OCDE (2006), la competencia científica incluye los conocimientos científicos y el uso que de esos conocimientos haga un individuo para plantear preguntas idóneas, adquirir nuevos conocimientos, explicar los fenómenos científicos y sacar conclusiones basadas en evidencias sobre asuntos relacionados con el área de las ciencias. Las tres subcompetencias implicadas en la definición anterior podrían delimitarse de la siguiente manera: i) identificar asuntos o temas científicos; 2) explicar científicamente los fenómenos; y 3) usar la evidencia científica.

Por supuesto, estas cuestiones mencionadas pueden tratarse desde el punto de la didáctica de la ciencia. No son pocos los autores que han propuesto diferentes metodologías desde didácticas orientadas a implementar los procesos de solución de problemas en la enseñanza de las ciencias (Bennett y Lubben, 2006; Charpak, Léna y Quéré, 2006; Duschl, I997; King, 2012; Schawartz et al., 1997), hasta trabajos por proyectos y otras metodologías activas que requieren la implicación del alumno en la generación de conocimientos. El objetivo de este trabajo no es ahondar en tales metodologías, sino profundizar en el estudio de las habilidades necesarias y fundamentales en el proceso de solución de problemas científicos: la creatividad científica.

De forma intuitiva todos reconocemos el papel que juega la creatividad en los descubrimientos científicos. Casi por definición la investigación científica requiere de la creatividad para ir más allá del conocimiento y de las técnicas conocidas, todo 
ello es necesario para crear nuevas formas de entender la realidad. La resolución de los problemas científicos exige que los estudiantes exploren su repertorio de conocimientos, imaginen formas distintas de resolver dichos problemas y creen combinaciones novedosas del conocimiento. Por esta razón, la creatividad científica debería ser considerada como una parte importante dentro del pensamiento científico (Weiping y Philip, 2002).

La creatividad puede definirse como la habilidad para ofrecer productos que son a la vez novedosos (originales) y apropiados a la tarea; esto es, válidos (Sternberg y Lubart, 1995/1997; Hennessey y Amabile, 1999). En este sentido, la creatividad se define como la capacidad para apreciar posibilidades nuevas y explotarlas. Implica un proceso mediante el cual se analizan los problemas e ideas desde nuevas perspectivas, porque la solución exige cambios e ideas brillantes y no convencionales (Ruiz, Bermejo, Ferrando, Prieto y Sainz, 20I4). En lo que se refiere al pensamiento creativo, éste ha sido caracterizado como 'pensamiento ordinario' (como opuesto a una forma extraordinaria de pensamiento) (Perkins, 2000/2003; Weisberg, 20I4), mientras que otros han defendido la existencia de procesos de pensamiento específicos que implican 'insight' (Sternberg y Davidson, 1999).

Los factores que influyen en la creación de productos novedosos y valiosos han sido estudiados por distintos autores: abarcando desde las características personales (inteligencia, motivación por la tarea, conocimiento previo y estilos intelectuales, entre otros) hasta las contextuales (oportunidades, el dominio concreto en el que se trabaja y el campo de expertos de ese dominio) (Csikszentmihalyi, 1996/1998; Sternberg y Lubart, 1995/1997).

Lin, Hu, Adey y Shen (2003) defienden la existencia de una creatividad específica de ciencias necesaria en la resolución de problemas, la generación de hipótesis, el diseño de experimentos y la innovación técnica, lo que requiere de una creatividad que es específica de las ciencias.

¿Por qué hablar de una creatividad específica en ciencias? Seguramente, como afirma Dunbar (1999), la mayoría de los investigadores piensan que la creatividad científica se compone de los mismos procesos mentales que guían cualquier otra forma de creatividad, y lo que hace a las ciencias diferentes es el vasto conocimiento (teórico, técnico y experimental) que se requiere para crear una idea novedosa.

En la literatura se ha aceptado que la creatividad en todas las disciplinas, especialmente en las ciencias, es de dominio específico (Baer, I998; Han, 2003). Esta especificidad puede que no venga especialmente marcada por los procesos mentales necesarios para crear nuevas ideas, sino por los conocimientos previos necesarios o por una mezcla de ambos (Plucker y Beghetto, 2004). Lo que sí se ha podido comprobar es que las personas pueden ser muy creativas en un dominio como en literatura y ser poco creativas en otro, como la música (Baer, 1999).

\section{Diferencias de género y creatividad científica}

Como hemos mencionado anteriormente, la creatividad no surge en el vacío, sino que se nutre de las experiencias y los conocimientos previos. Es por ello que el contexto sociocultural en el que se desarrollan los estudiantes influye en el interés que chicos y chicas manifiestan hacia las ciencias. Algunos estudios indican que hay profesiones, especialmente dentro del área científica, que se asocian tradicionalmente al 
género masculino (Diekman, Brown, Johnston y Clark, 20ıo). Esta concepción puede contribuir a que las mujeres tiendan a preferir carreras en las que tengan la posibilidad de trabajar o de ayudar a otras personas (Halpern et al., 2007).

Más allá del contexto sociocultural, la característica idiosincrásica del pensamiento creativo es el pensamiento divergente. Se han llevado a cabo distintos estudios sobre diferencias de género en el pensamiento divergente, dichos estudios llegan a resultados no consistentes y, por tanto, no podemos decir que estas diferencias de género sean generalizables (para una revisión consúltese Baer y Kaufman, 2008). De los estudios más recientes que han investigado las diferencias de género en creatividad científica comentamos brevemente los más relevantes.

Mohamed (2006) realiza un estudio con 138 alumnos de quinto grado de seis escuelas diferentes. En este trabajo se utiliza un Test de Creatividad Científica compuesto por tres subtest: I) Problemas y Soluciones; 2) Agrupamiento de flores; y 3) Diseño de un experimento. Estas tareas contienen varios ítems que evalúan las dimensiones de la creatividad: fluidez, flexibilidad y originalidad. En cuanto a las propiedades psicométricas obtenidas, cabe destacar un alfa de Cronbach de .90 para el conjunto de la prueba; mientras que el subtest I obtuvo un $a=.82$; el subtest 2 un a $=.8 \mathrm{I} ; \mathrm{y}$ el subtest 3 un a $=.9$ I. En este estudio se analizaron las diferencias de género utilizando un modelo lineal general. Se comprobó que el efecto del género para la interacción global de los tres subtest no era significativo. Sin embargo, las chicas fueron significativamente superiores a los chicos en los subtest 2 y 3 .

Jo (2009) hizo un estudio sobre creatividad y conocimiento científico. Se utilizó una muestra de 295 alumnos de secundaria de nacionalidad coreana, con edades comprendidas entre los I2 y los I4 años. Para medir la creatividad en ciencias el autor usó una tarea abierta con el formato "que pasaría si...». La tarea fue evaluada en términos de fluidez, flexibilidad, originalidad, elaboración y transformación. Los datos procedentes de este estudio no arrojaron diferencias significativas entre chicos y chicas.

En el trabajo de Okere y Ndeke (20I2) se estudió la relación entre el conocimiento científico y la creatividad en el área de biología. En su estudio se incluyó el análisis de diferencias de género. Para ello, los autores aplicaron dos pruebas a 363 estudiantes de ocho centros de Nakuru (Kenia). Una de las pruebas estaba dirigida a evaluar el nivel de conocimiento en biología de los estudiantes y la otra a estudiar el nivel de habilidad en creatividad científica en biología (valora cuatro dimensiones de la creatividad: sensibilidad; reconocimiento de relaciones; planificación de la investigación; y flexibilidad en el razonamiento). A partir de los datos obtenidos concluyeron lo siguiente: la creatividad científica en biología era superior en los chicos que en las chicas; incluso los chicos fueron superiores en las dimensiones referidas a la flexibilidad, reconocimiento de relaciones y sensibilidad; sin embargo, no se encontraron diferencias entre chicos y chicas en la dimensión de planificación.

Un estudio similar lo presentan Ayverdi, Asker, Öz Aydin y Saritaş (20ı2), utilizando también el test de $\mathrm{Hu}$ y Adey (2002). Los participantes fueron 145 alumnos de educación primaria (de sexto a octavo grado) de nacionalidad turca. Los datos encontrados por estos autores indicaron que no existían diferencias estadísticamente significativas entre chicos y chicas en creatividad científica.

Ruiz (2013) llevó a cabo un estudio con una muestra de 98 estudiantes de Educación Secundaria de nacionalidad española, a quienes se les administró la prueba de Hu y Adey (2002). Dicha prueba había sido adaptada a nuestro contexto y se obtuvo 
un alfa de Cronbach de .899 (Ruiz, Bermejo, Prieto, Ferrándiz y Almeida, 2013). Los resultados hallados por Ruiz (2013) mostraron que las chicas obtuvieron puntuaciones más elevadas que los chicos en todas las tareas de la prueba, pero estas diferencias no fueron estadísticamente significativas. Esto apunta a que las chicas muestran mayor número de respuestas a la hora de solucionar las distintas tareas (fluidez de ideas); manifiestan mayor número de ideas diferentes (flexibilidad); e incluso sus respuestas son más inusuales y novedosas (originalidad) que las de los chicos de esta muestra.

Ceran, Güngören y Boyacioğlu (2014), utilizando también el test de $\mathrm{Hu}$ y Adey (2002) en una muestra de 227 estudiantes de Educación Secundaria de nacionalidad turca, corroboraron la no existencia de diferencias de género.

Utilizando una prueba distinta, Özdemir y Sak (2013) llevaron a cabo un estudio en el que participaron 704 estudiantes ( 359 chicos) de sexto grado identificados como superdotados y de alto rendimiento. Todos ellos asistían al programa EPTs (Education Programs for Talented Students) para niños superdotados ofrecido por la Anadolu University (Turquía). En dicho estudio se utilizó el C-SAT (Test de Habilidad de la Creatividad Científica, Sak y Ayas, 2orI), el cual valora distintos componentes de la creatividad científica (generación de hipótesis, diseño de experimentos y evaluación de la evidencia), así como las dimensiones de la creatividad (fluidez, flexibilidad y compuesto creativo). En relación a las propiedades psicométricas del C-SAT cabe destacar una adecuada fiabilidad $(a=.848)$. Los resultados mostraron que los chicos puntuaron más alto en todos los componentes de la creatividad científica (generación de hipótesis, diseño de experimentos y evaluación de la evidencia); es decir, mostraban mayor fluidez a la hora de proponer posibles hipótesis para la solución de problemas; asimismo, los chicos mostraban mayor flexibilidad a la hora de modificar los experimentos para comprobar una hipótesis; e incluso mostraron una cierta superioridad cuando tenían que evaluar las evidencias propuestas en las tareas. Los autores concluyeron que las diferencias fueron estadísticamente significativas a favor de los chicos en la generación de hipótesis, en fluidez y en el compuesto creativo.

El análisis de estas investigaciones refleja el debate en relación a las diferencias de género. En este sentido, cuando se utilizan pruebas que no tienen una carga de conocimiento específico en ciencias (como es el test de Hu y Adey, 2002, o la prueba diseñada por Mohamed, 2006), no se encuentran diferencias estadísticamente significativas entre chicos y chicas. Sin embargo, cuando la prueba requiere un conocimiento en las áreas específicas de ciencias (biología, ecología, física y química), se observa que los chicos tienen mayores destrezas en la creatividad científica.

\section{Diferencias en creatividad científica según nivel educativo}

Tal y como hemos indicado, el conocimiento previo en las ciencias es un factor importante a la hora de tener un acopio de ideas que puedan combinarse para dar lugar a nuevos planteamientos (Aktamis y Ergin, 2008; Jo, 2009; Liang, 2002). En este sentido, el nivel educativo de los participantes podría tomarse como un cierto indicador del conocimiento asimilado.

Las investigaciones que se han centrado en estudiar el rol del conocimiento previo encuentran que sí existen diferencias en función del curso o nivel académico (Ayverdi et al., 2012; Ceran et al., 2014; Ruiz, 2013). 
En el estudio realizado por Ayverdi et al. (20I2) se encontraron diferencias estadísticamente significativas dependiendo del curso académico. Así, los alumnos de séptimo grado puntuaban significativamente por encima de sus compañeros de sexto y octavo en la prueba de Hu y Adey (2002). Las puntuaciones superiores de los estudiantes de séptimo con respecto a los de octavo podrían explicarse por el descenso en creatividad en la edad preadolescente; este dato se ha reportado en la literatura y se conoce como el fenómeno del 4th grade slump (Ferrando y Ferrándiz, 2013).

En el estudio de Ruiz (2013) se encontraron diferencias estadísticamente significativas en todas las tareas del test de Hu y Adey (2002), estas diferencias fueron a favor de los estudiantes de $4 .^{\circ}$ de la EsO. Es decir, que los estudiantes de los cursos superiores obtienen mejores rendimientos en las tareas de creatividad científica.

En el trabajo de Ceran et al. (20I4) se indicaron diferencias estadísticamente significativas en función del curso académico; es decir, los estudiantes de los últimos cursos de Educación Secundaria obtuvieron mayores puntuaciones, lo que indica que el conocimiento y el mayor nivel de experiencia influyen en la creatividad científica.

\section{Método}

\section{Participantes}

En el estudio han participado 78 estudiantes (40 chicos) de Educación Secundaria Obligatoria (ESO). Todos ellos con edades comprendidas entre I2 y los I6 años $(M=$ I3.70; $D T=$ I.27). Estos estudiantes han sido identificados como alumnos de altas habilidades (superdotados y talentos), siguiendo el procedimiento propuesto por Castelló y Batlle (I998). La Tabla I muestra una distribución de los participantes según su sexo y curso académico.

Tabla I. Distribución de los participantes en función del sexo y del curso

\begin{tabular}{|c|c|c|c|c|c|}
\hline & \multicolumn{5}{|c|}{ CURSO } \\
\hline & I. $^{\circ} \mathrm{ESO}$ & $2 .{ }^{\circ} \mathrm{ESO}$ & $3 .{ }^{\circ} \mathrm{ESO}$ & $4 .^{\circ} \mathrm{ESO}$ & TOTAL \\
\hline CHICOS & $\mathrm{I} 3$ & $\mathrm{IO}$ & 9 & 8 & 40 \\
\hline CHICAS & $\mathrm{I} 8$ & 6 & 5 & 9 & 38 \\
\hline TOTAL & $3 \mathrm{I}$ & $\mathrm{I} 6$ & $\mathrm{I} 4$ & $\mathrm{I} 7$ & 78 \\
\hline
\end{tabular}

\section{Instrumento}

En este trabajo se utiliza el C-SAT (Creative Scientific Ability Test: Test de Habilidad de la Creatividad Científica, Sak y Ayas, 20II). El test se ha diseñado para alumnos de Educación Secundaria. El tiempo aproximado de aplicación es de 40 minutos.

Los autores del test entienden que la creatividad se puede considerar como un proceso de interacción entre las habilidades generales de la creatividad, las habilidades relacionadas con el conocimiento científico y el conocimiento de las distintas áreas de ciencias. El test está fundamentado en una teoría sólida sobre la creatividad 
científica, que incluye tres componentes: las habilidades generales de la creatividad (fluidez, flexibilidad y un compuesto creativo); las habilidades relacionadas con las ciencias (generación de hipótesis, evaluación de las hipótesis a través del diseño de experimentos y evaluación de la evidencia); y el conocimiento que los alumnos tienen de las distintas áreas de ciencias (biología, física, química y ecología). El c-sAT está compuesto por cinco subtest que evalúan las habilidades científicas que los alumnos utilizan a través de 5 tareas en las que tienen que plantear hipótesis, crear experimentos para poder probar una determinada hipótesis y evaluar una evidencia que se les proporciona. Para una mayor comprensión de las tareas, se les da a los estudiantes dibujos ilustrativos de la actividad que han de realizar, lo que facilita los procesos de generación que se van a valorar y puntuar. Estos subtest se recogen en la Tabla 2. Cada tarea se puntúa por fluidez y por flexibilidad (categorías distintas utilizadas) y de cada tarea se calcula un Compuesto Creativo. Este compuesto creativo se calcula a través del logaritmo propuesto por Snyder, Mitchell, Bossomaier y Pallier (2004), el cual combina las puntuaciones de fluidez y flexibilidad. Esta prueba presenta unas adecuadas propiedades psicométricas, obteniendo un alfa de Cronbach de 848 (Sak y Ayas, 2013). En el presente estudio se ha verificado la estructura interna de un único componente, que explica el $38.93 \%$ de la varianza y cuya fiabilidad es $a=.680$ para los cinco elementos que componen la prueba.

Tabla 2. Subtest del Test de Creatividad Científica de Habilidad (C-SAT) tomado de Sak \& Ayas (20II)

\begin{tabular}{|l|l|l|l|l|l|}
\hline & \multicolumn{5}{|c|}{ Subtests } \\
\hline Tareas & $\begin{array}{l}\text { Experimento } \\
\text { de Vuelo }\end{array}$ & $\begin{array}{l}\text { Gráfico de } \\
\text { Interacción }\end{array}$ & $\begin{array}{l}\text { Experimento de } \\
\text { Azúcar }\end{array}$ & $\begin{array}{l}\text { Experimento del } \\
\text { Muelle }\end{array}$ & $\begin{array}{l}\text { Cadena } \\
\text { Alimentaria }\end{array}$ \\
\hline Área de Ciencias & Biología & $\begin{array}{l}\text { Interdiscipli- } \\
\text { nariedad }\end{array}$ & Química & Física & Ecología \\
\hline Proceso Científico & $\begin{array}{l}\text { Generación de } \\
\text { Hipótesis }\end{array}$ & $\begin{array}{l}\text { Generación de } \\
\text { Hipótesis }\end{array}$ & $\begin{array}{l}\text { Evaluación de } \\
\text { Hipótesis }\end{array}$ & $\begin{array}{l}\text { Evaluación de } \\
\text { Hipótesis }\end{array}$ & $\begin{array}{l}\text { Evaluación de la } \\
\text { Evidencia }\end{array}$ \\
\hline Habilidades Creativas & $\begin{array}{l}\text { - Fluidez } \\
\text { - Flexibilidad } \\
\text {-Composición } \\
\text { creativa }\end{array}$ & $\begin{array}{l}\text { - Fluidez } \\
\text { - Flexibilidad } \\
\text {-Composición } \\
\text { creativa }\end{array}$ & $\begin{array}{l}\text { - Fluidez } \\
\text {-Flexibilidad } \\
\text {-Composición } \\
\text { creativa }\end{array}$ & $\begin{array}{l}\text { - Fluidez } \\
\text { - Flexibilidad } \\
\text {-Composición } \\
\text { creativa }\end{array}$ & $\begin{array}{l}\text { - Fluidez } \\
\text { - Flexibilidad } \\
\text {-Composición } \\
\text { creativa }\end{array}$ \\
\hline
\end{tabular}

\section{Procedimiento}

El C-SAT fue adaptado por diferentes miembros del Equipo de Altas Habilidades de la Universidad de Murcia, siguiendo las normas de la International Test Commision (Hambleton, Merenda y Spielberger, 2005).

Posteriormente, se administró a los alumnos en horario lectivo. Al principio de la sesión se leyeron todas las preguntas a los estudiantes, para resolver las posibles dudas que les pudieran surgir; y después, se les dejó 40 minutos para realizar la prueba.

Una vez que la prueba se había aplicado, se transcribieron las respuestas de los alumnos a una base de datos utilizando el programa EXCEL, con el fin de que la grafía de los alumnos no influyese en la puntuación de sus respuestas. La corrección de la prueba se realizó por personas formadas para tal fin. 
Por último, se realizaron los análisis estadísticos con el programa sPss 20 (IBM Corp., 20II).

\section{Análisis de datos}

Se llevaron a cabo diferentes análisis utilizando distintos procedimientos dentro de un método general de carácter descriptivo. Las técnicas de análisis fueron: a) análisis descriptivos de las puntuaciones obtenidas por los participantes en función del género y del curso académico, se utilizaron los coeficientes media y desviación típica; y b) análisis de diferencia de medias para muestras independientes, realizado mediante prueba t y ANOVA.

\section{Resultados}

La Tabla 3 presenta los estadísticos descriptivos de las puntuaciones de chicos y chicas en las distintas dimensiones valoradas por la prueba y en cada una de las tareas. Según muestran las medias, se puede apreciar que las puntuaciones de ambos son bastante similares; sin embargo, los chicos destacan más que las chicas en la tarea I (generación de hipótesis en el área de biología); también en la tarea 2 (generación de hipótesis en diferentes disciplinas); en el proceso de generación de hipótesis, que se valora a través de ambas tareas; e incluso en el total de la prueba. Mientras que las chicas muestran mayor habilidad en la tarea 4 (evaluación de hipótesis en el área de física); en la tarea 5 (evaluación de las evidencias en el área de ecología); y en la evaluación de hipótesis, que está incluida en las tareas 3 y 4.

Figura i. Gráfico de las puntuaciones medias obtenidas para cada tarea en función del género

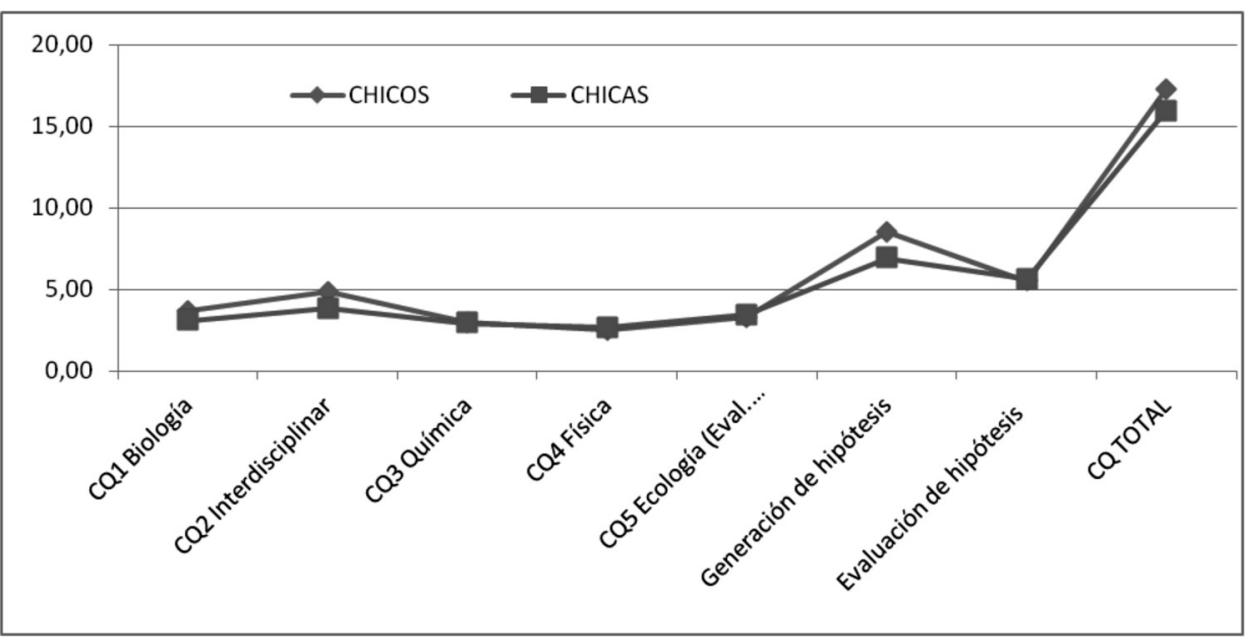


Para comprobar si estas diferencias son estadísticamente significativas, se procedió a realizar un análisis de comparación de medias (t de Student). Los resultados de dicho análisis mostraron que las diferencias eran significativas a favor de los chicos, para la generación de hipótesis (incluidas en las tareas i y 2) y para la tarea 2.

Tabla 3. Estadísticos descriptivos, puntuaciones t de Student y la potencia observada de las puntuaciones de compuesto creativo de las distintas tareas del C-SAT

\begin{tabular}{|c|c|c|c|c|c|c|c|c|}
\hline & \multicolumn{3}{|c|}{ Chicos $(\mathrm{N}=40)$} & \multicolumn{3}{|c|}{ Chicas $(\mathrm{N}=38)$} & \multirow[b]{2}{*}{ T de student } & \multirow[b]{2}{*}{$\begin{array}{l}\text { Potencia } \\
\text { observada }\end{array}$} \\
\hline & Mín.-Máx. & Media & $\mathrm{Dt}$ & Mín.-Máx. & Media & Dt & & \\
\hline CQi Biología & $0-7,58$ & 3,66 & $\mathrm{I}, 9 \mathrm{I}$ & $0-7,75$ & 3,IO & 2,24 & $\mathrm{~T}(76)=\mathrm{I}, \mathrm{I} 88 ; \mathrm{p}=, 239$ & ,216 \\
\hline $\mathrm{CQ}_{2}$ Interdisciplinar & $0-8,75$ & 4,84 & 2,09 & $0-7,75$ & 3,83 & I,96 & $\mathrm{T}(76)=2,215 ; \mathrm{p}=, 030$ &, 590 \\
\hline $\mathrm{CQ}_{3}$ Química & $0-6,58$ & 2,99 & 1,96 & $0-6,75$ & 2,94 & $\mathrm{I}, 83$ & $\mathrm{~T}(76)=, 099 ; \mathrm{p}=, 92 \mathrm{I}$ & ,05I \\
\hline $\mathrm{CQ}_{4}$ Física & $0-6,17$ & 2,53 & $\mathrm{I}, 6 \mathrm{I}$ & $0-6,58$ & 2,66 & $\mathrm{I}, 77$ & $\mathrm{~T}(76)=-, 350 ; \mathrm{p}=, 727$ & ,064 \\
\hline $\begin{array}{l}\text { CQ5 Ecología } \\
\text { (Eval. Evidencia) }\end{array}$ & $0-8,17$ & 3,29 & 1,96 & $0-7,49$ & 3,43 & 1,76 & $\mathrm{~T}(76)=-, 350 ; \mathrm{p}=, 727$ & ,064 \\
\hline Generación de hipótesis & $3-14,49$ & 8,50 & 2,72 & $\mathrm{I}-\mathrm{I} 3,75$ & 6,93 & 2,99 & $\mathrm{~T}(76)=2,439 ; \mathrm{p}=, \mathrm{oI} 7$ & 673 \\
\hline Evaluación de hipótesis & $\mathrm{I}-\mathrm{I} 2, \mathrm{I} 7$ & 5,52 & 2,89 & $0-12, I 7$ & $5,6 \mathrm{I}$ & 2,98 & $\mathrm{~T}(76)=-, \mathrm{I} 37 ; \mathrm{p}=, 89 \mathrm{I}$ & ,052 \\
\hline CQ TOTAL & $6,58-28,89$ & $\mathrm{I} 7,3 \mathrm{I}$ & 5,22 & $4-27,66$ & 15,97 & 6,40 & $\mathrm{~T}(76)=\mathrm{I}, 013 ; \mathrm{p}=, 314$ & , I70 \\
\hline
\end{tabular}

\section{Diferencias según curso académico}

En primer lugar, hemos hallado los estadísticos descriptivos para cada curso. Los cuales se muestran en la Tabla 4 y de forma más intuitiva en la Figura 2. Como se observa, los estudiantes que mejores puntuaciones obtienen son los de $2 .^{\circ} \mathrm{y} 4 .^{\circ}$ de la ESO. Los estudiantes de segundo destacan especialmente en la tarea 2 (contenido interdisciplinar) y en la tarea 4 (contenido de física), así como en la generación de hipótesis. Mientras que los estudiantes de cuarto son quienes mayores puntuaciones obtienen en el total de la prueba, en la tarea i (contenido de biología), en la tarea 3 (contenido de química) e incluso en la tarea 5 (contenido de ecología). Son los estudiantes de primero quienes obtienen menores puntuaciones. 
Figura 2. Gráfico de puntuaciones medias obtenidas para cada una de las tareas en función del curso

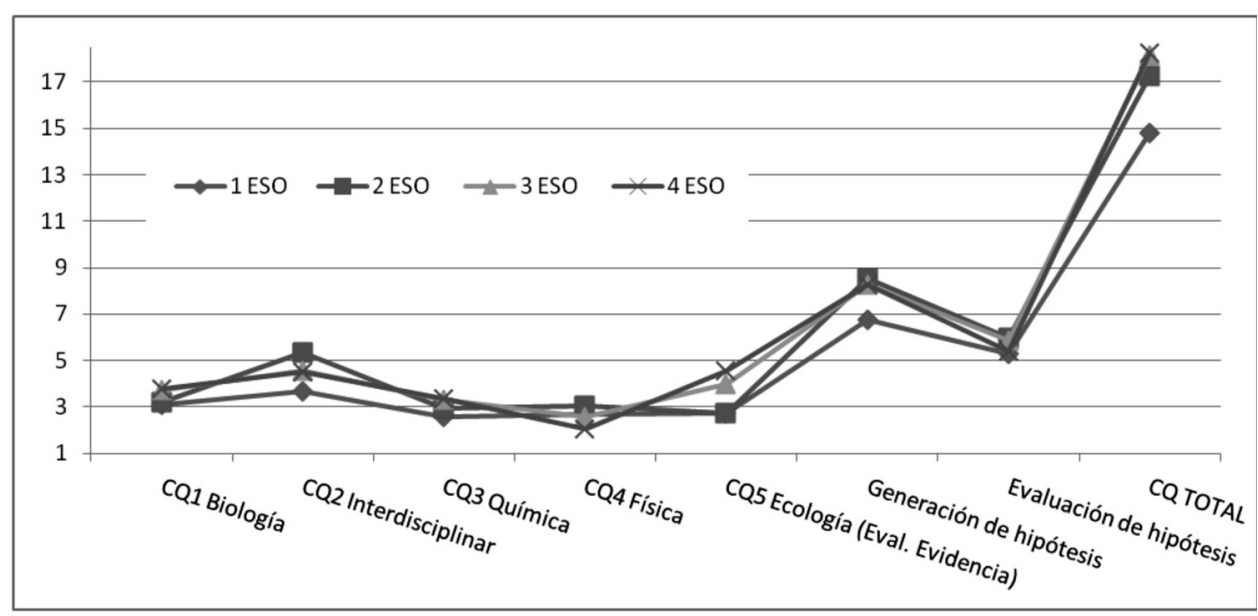

Para conocer si estas diferencias eran estadísticamente significativas, se procedió a realizar un análisis de varianzas (ANOvA). Los resultados procedentes de este análisis indicaron que las diferencias eran estadísticamente significativas sólo para la tarea 5 $[\mathrm{F}(3,74)=5,508 ; p=.002]$. Los análisis post-hoc mostraron que las diferencias en la tarea 5 se daban entre los estudiantes de $4 .^{\circ}$ y los de I. ${ }^{\circ}$ y $2 .^{\circ}$, siempre a favor de los estudiantes de $4 .^{\circ}$ de la ESO. También se encontraron diferencias marginalmente significativas para la tarea $2[\mathrm{~F}(3,74)=2,507 ; p=.065]$; los análisis post-hoc mostraron que estas diferencias se encontraban entre los estudiantes de I. ${ }^{\circ}$ y $2 .^{\circ}$ de la EsO, a favor de estos últimos.

Tabla 4. Estadísticos descriptivos por curso y resultados de las comparaciones post-hoc en los casos en los que se encontraron diferencias estadísticamente significativas

\begin{tabular}{|c|c|c|c|c|c|c|c|c|c|}
\hline & \multicolumn{2}{|c|}{ I. ${ }^{\circ} \mathrm{ESO}$} & \multicolumn{2}{|c|}{ 2. ${ }^{\circ} \mathrm{ESO}$} & \multicolumn{2}{|c|}{$3 .{ }^{\circ} \mathrm{ESO}$} & \multicolumn{2}{|c|}{$4 .^{\circ} \mathrm{ESO}$} & \multirow{2}{*}{ Post-hoc } \\
\hline & $\mathrm{M}$ & DT & $\mathrm{M}$ & DT & $\mathrm{M}$ & DT & $\mathrm{M}$ & DT & \\
\hline CQi Biología & $3, \mathrm{II}$ & $\mathrm{I}, 9 \mathrm{I}$ & $3,2 \mathrm{I}$ & 2,20 & 3,70 & 1,82 & 3,80 & $2,5 \mathrm{I}$ & \\
\hline $\mathrm{CQ}_{2}$ Interdisciplinar & 3,67 & $\mathrm{I}, 80$ & 5,32 & 1,62 & 4,58 & 2,OI & 4,49 & 2,66 & $2 .^{\circ}>\mathrm{I}^{\mathrm{o}}$ \\
\hline CQ3 Química & 2,60 & 1,87 & 2,93 & $\mathrm{I}, 5 \mathrm{I}$ & 3,31 & I, 77 & 3,38 & 2,30 & \\
\hline $\mathrm{CQ}_{4}$ Física & 2,68 & I,84 & 3,05 & $\mathrm{I}, 47$ & 2,57 & 1,57 & 2,03 & $\mathrm{I}, 63$ & \\
\hline CQs Ecología (Eval. Evidencia) & 2,75 & I,90 & $2,7 \mathrm{I}$ & $\mathrm{I}, 34$ & $4, \mathrm{OI}$ & 1,60 & 4,55 & $\mathrm{I}, 73$ & $4^{\circ} .^{\circ}>I^{\circ} ; 4^{\circ} .^{0}>2 .^{\circ}$ \\
\hline Generación de hipótesis & 6,77 & $2,8 \mathrm{I}$ & 8,53 & 2,97 & 8,28 & 2,34 & 8,29 & 3,33 & \\
\hline Evaluación de hipótesis & 5,28 & 3,27 & 5,98 & 2,38 & 5,88 & 2,6I & $5,4 \mathrm{I}$ & $3, \mathrm{II}$ & \\
\hline CQ TOTAL & 14,80 & 6,44 & 17,23 & 5,52 & $\mathrm{I8,17}$ & 4,17 & $\mathrm{I} 8,25$ & 5,58 & \\
\hline
\end{tabular}




\section{Discusión y conclusiones}

Tal y como hemos analizado anteriormente, podemos decir que el tema de la creatividad científica sigue siendo un tema candente en la actualidad. Especialmente hay una preocupación por el estudio de las diferencias de género y el nivel escolar. Otra de las preocupaciones que tienen ahora los investigadores es responder al bajo número de chicas que se implican en las carreras científicas, incluso cuando manifiestan habilidades y conocimientos para poder cursarlas. De ahí la importancia de este trabajo.

Según se desprende de la revisión realizada se han encontrado evidencias que afirman que los chicos alcanzan puntuaciones superiores a las chicas en el área de las ciencias (Özdemir y Sak, 20I3) y también a la inversa; es decir, las chicas manifiestan mayor nivel e interés por el área científica y por las actividades sociales (Lubart y Zenasni, 20Io); incluso que no hay diferencias en función del género en la creatividad científica (Ayverdi et al., 2012; Baer y Kaufman, 2008).

Con el fin de seguir ahondando en esta problemática se ha utilizado una prueba de creatividad científica que implica el manejo de un cierto nivel de conocimientos en distintas áreas de ciencias, para estudiar posibles diferencias de género y nivel educativo.

Los resultados encontrados en nuestro trabajo apuntan a que los chicos obtienen mejores rendimientos que las chicas en la creatividad científica y en el dominio de las ciencias (biología, física, química y ecología); estos datos procedentes de nuestra investigación coinciden con los hallados por Özdemir y Sak (2013).

La superioridad de los chicos en las tareas i y 2 indica una mayor capacidad para generar diferentes hipótesis sobre problemas en diferentes áreas (ecología, economía, salud y social), cuya solución exige grandes dosis de fluidez y flexibilidad (Özdemir y Sak, 2013). Estos resultados se verifican en el estudio de Okere y Ndeke (20I2). A esto hay que añadir que la flexibilidad es una característica propia de personas creativas que dominan un campo de conocimiento (Sternberg y O'Hara, 2005).

En las tareas 4 y 5 , las chicas obtienen mejores rendimientos; ambas tareas exigen evaluación de hipótesis y de evidencias en las áreas de física y ecología, respectivamente. Aunque las diferencias entre chicas y chicos no son significativas, sí que hay una cierta superioridad de las chicas frente a los chicos, datos que no coinciden con los resultados hallados por Özdemir y Sak (2013). Sin embargo, nuestros datos estarían más acordes con los hallados por otros autores, quienes indican la superioridad de las chicas en el pensamiento divergente (Ayverdi et al., 20I2; Ceran et al., 20I4; Ruiz, 2013).

En general, podemos decir que los chicos de nuestro estudio siguen siendo superiores a las chicas en la creatividad científica, datos que concuerdan con los hallados por otros autores (Özdemir y Sak, 20ı3).

En relación con el curso o nivel educativo, nuestros datos indican que los estudiantes de cursos superiores (chicos y chicas) puntúan más alto en algunas de las dimensiones del C-SAT, pero estas diferencias sólo son significativas en la tarea 5; este resultado concuerda con el obtenido por Ruiz (2013), quien encontró que los estudiantes de cursos superiores puntuaban más alto en las prueba de Hu y Adey (2002). Estos datos señalarían la influencia que tiene el manejo de conocimientos científicos en la capacidad para evaluar una evidencia dada. Estos datos concuerdan con los hallados por Okere y Ndeke (20I2), quienes señalaron la relación existente entre el conocimiento en el área de biología y la creatividad científica. 
A la luz de estos resultados podemos establecer dos grandes conclusiones.

Primera, tenemos que destacar que en este estudio se ha utilizado un test novedoso donde se incluyen conocimientos de las diferentes áreas de las ciencias, habilidades propias del científico y las principales de la creatividad. Esto indica un alto grado de flexibilidad, característica esencial de la creatividad. Esta capacidad permite explorar, generar hipótesis, modificarlas, transformarlas para adaptarlas a la solución más ingeniosa.

Segunda, queremos señalar que en futuras investigaciones deberíamos controlar los efectos del conocimiento previo, rendimiento académico y del pensamiento divergente de dominio general sobre la creatividad científica.

Tercera, tras lo expuesto anteriormente, se podría destacar que el hecho de que las diferencias entre los alumnos de los cursos superiores e inferiores se encuentre en la tarea 5 (referente al área de ecología), se podría percibir como una mayor influencia del conocimiento del estudiante a la hora de resolverla de forma novedosa e incluyendo gran cantidad de posibles soluciones ante la evidencia planteada. En este sentido, por un lado, cabría destacar que el contenido de esta tarea se encuentra más próximo a los contenidos curriculares y, por otro lado, que las demás tareas no se ciñen a los contenidos incluidos en el currículo ordinario. Por todo ello, se podría concluir que quizá en las tareas referentes a las áreas de biología, física y química exista un mayor peso del nivel de habilidad para las ciencias y de creatividad.

Finalmente, hay que añadir que en estudios posteriores es necesario ampliar la muestra de participantes, porque nos permitirá realizar otro tipo de análisis de covarianza en el que pudieran estudiarse los efectos combinados de las variables curso y género.

\section{Bibliografía}

Aktamis, H. y Ergin, Ö. (2008) The effect of scientific process skills education on students scientific creativity, science attitudes and academic achievements. Asia-Pacific Forum on Science Learning and Teaching, 9 (I), I-2I.

Ayverdi, L.; Asker, E.; Öz Aydin, S. y Saritaș, T. (20I2) Determination of the relationship between elementary students' scientific creativity and academic achievement in science and technology courses. Illköğretim Online, II, 646-659. Obtenido de: http://ilkogretim-online. org.tr/volinsay3/viss3m6.pdf.

BAER, J. (1998) The case for domain specificity of creativity. Creativity Research Journal, II (2), I73-177.

http://dx.doi.org/Io.I207/si5326934crjiro2_7

Baer, J. (1999) Domains of creativity. En M. A. Runco y S. Pritzker (eds.) Encyclopedia of Creativity (pp. 591-596). New York: Academic Press.

Baer, J. y Kaufman, J. C. (2008) Gender Differences in Creativity. The Journal of Creative Behaviour, 42 (2), 75-105. http://dx.doi.org/I0.1002/j.2162-6057.2008.tbor289.x

Bennett, J. y Lubben, F. (2006) Context based chemistry: The Salters approach. International Journal of Science Education, 28 (9), 999-I0I5. http://dx.doi.org/I0.I080/09500690600702496

Castelló, A. y Batlle, C. (1998) Aspectos teóricos e instrumentales en la identificación del alumnado superdotado y talentoso. Propuesta de un protocolo. FAISCA, 6, 26-66.

Ceran, S. A.; GÜNGören, S. Ç. y BoYACIOĞLU, N. (2OI4) Determination of scientific creativity levels of middle school students and perceptions through their teachers. European Journal of Research on Education, 47-53. Obtenido de: http://iassr.org/rs/020408.pdf. 
Charpak, G.; LÉna, P. y Quéré, Y. (2006) Los niños y la ciencia: La aventura de la mano en la masa. Siglo XXI.

Csikszentmihalyi, M. (1996/1998) Creativity: Flow and the Psychology of Discovery and Invention. New York: Harper. Trad. castellano: Creatividad: el Fluir y la Psicología del Descubrimiento y la Invención. Barcelona: Paidós Ibérica.

Diekman, A. B.; Brown, E. R.; Johnston, A. M. y Clark, E. K. (20io) Seeking congruity between goals and roles a new look at why women opt out of science, technology, engineering, and mathematics careers. Psychological Science, 2I (8), I05I-I057. http://dx.doi.org/ı.1177/0956797610377342

Dunbar, K. (1999) Science. En M. A. Runco y S. R. Pritzker (eds.) Encyclopedia of Creativity (vol. II) (pp. 525-53I). New York: Elsevier.

Duschl, R. A. (1997) Renovar la enseñanza de las ciencias: importancia de las teorias y su desarrollo. Madrid: Narcea.

Ferrando, M. y Ferrándiz, C. (2013) Early years' Creativity. En A. Gariboldi y N. CateLLANI (eds.) Creativity in Preschool Education (pp. 70-78). Scandiano, Italia: SERN. (ISBN-I3: 978-8890487842).

Halpern, D. F.; Benbow, C. P.; Geary, D. C.; Gur, R. C.; Hyde, J. S. y Gernsbacher, M. A. (2007) The science of sex differences in science and mathematics. Psychological science in the public interest, 8 (I), I- $5 \mathrm{I}$.

http://dx.doi.org/IO.IIII/j.I529-IO06.2007.00032.x

Hambleton, R. K.; Merenda, P. y Spielberger, C. (eds.) (2005) Adapting educational and psychological tests for cross-cultural assessment. Hillsdale, NJ: Lawrence S. Erlbaum Publishers.

Han, K. S. (2003) Domain Specificity of Creativity in Young Children: How Quantitative and Qualitative Data Support It. The journal of creative behavior, 37 (2), II7-I42. http://dx.doi.org/I0.I002/j.2162-6057.2003.tboo829.x

Hennessey, B. A. y Amabile, T. M. (1999) Consensual assessment. En M. A. Runco y S. R. Pritzker (eds.) Encyclopedia of Creativity (vol. I) (pp. 347-359). New York: Elsevier.

Hu, W. y Adey, P. A. (2002) Scientific creativity test for secondary school students. International Journal of Science Education, 24 (4), 389-403. http://dx.doi.org/I0.1080/09500690110098912

IBM Corp. (20II) IBM SPSS Statistics for Windores, Version 20.0. Armonk, NY: IBM Corp.

Jo, S. M. (2009) A study of Korean students' creativity in science using structural equation modeling. Unpublished $\mathrm{PhD}$ thesis. The University of Arizona.

KING, D. (2OI2) New perspectives on context-based chemistry education: using a dialectical sociocultural approach to view teaching and learning. Studies in Science Education, 48 (I), 5I-87. http://dx.doi.org/Io.1080/03057267.2012.655037

LIANG, J. (2002) Exploring scientific creativity of eleventh grade students in Taiwan. Unpublished PhD thesis. The University of Texas at Austin.

Lin, C.; Hu, W.; Adey, P. y Shen, J. (2003) The influence of CASE on scientific creativity. Research in Science Education, 33, I43-162. DOI: I0.1023/A: 1025078600616.

Lubart, T. y Zenasni, F. (20I0) A New Look at Creative Giftedness. Gifted and Talented International, $25(\mathrm{I}), 53-57$.

Mohamed, A. (2006) Investigating the Scientific Creativity of fifth-grade students. Unpublished PhD Thesis. The University of Arizona, Tucson, Arizona.

OCDE (2006) El programa PISA de la OCDE ¿Qué es y para qué sirve? París: OCDE. Recuperado en http://www.oecd.org/pisa/39730818.pdf (última visita 2015.03.05).

Okere, M. I. y Ndeke, G. C. W. (20I2) Influence of gender and knowledge on secondary school students' scientific creativity skills in Nakuru District, Kenya. European Journal of Educational Research, I (4), 353-366.

Özdemir, N. N. y SAK, U. (2OI3) A Componential Analysis of Gender Differences in Scientific Creativity. Turkish Journal of Giftedness and Education, 3 (2), 53-65. 
Perkins, D. N. (2000/2003) Archimedes' bathtub: The art and logic of breakthrough thinking. New York: Norton \& Company. Trad. castellano: La bañera de Arquimedes y otras historias del descubrimiento científico: el arte del pensamiento creativo. Barcelona: Paidós.

Plucker, J. A. y Beghetto, R. A. (2004) Why Creativity Is Domain General, Why It Looks Domain Specific, and Why the Distinction Does Not Matter. En R. J. STernberg, E. L. Grigorenko y J. L. Singer (eds.) Creativity: From potential to realization (pp. I53-167). Washington, DC, us: American Psychological Association. http://dx.doi.org/Io.IO37/I0692-009

Ruiz, M. J. (2013) Estudio del pensamiento cientifico-creativo en una muestra de alumnos de Educación Secundaria. Trabajo Fin de Máster. Murcia: Universidad de Murcia.

Ruiz, M. J.; Bermejo, R.; Ferrando, M.; Prieto, M. D. y Sainz, M. (20I4) Inteligencia y Pensamiento Científico-Creativo: Su convergencia en la explicación del rendimiento académico de los alumnos. Electronic Journal of Research in Educational Psychology, I2 (2), 283-302.

Ruiz, M. J.; Bermejo, M. R.; Prieto, M. D.; Ferrándiz, C. y Almeida, L. S. (20I3) Evaluación del Pensamiento Científico-Creativo: Adaptación y validación de una prueba en población española. Revista Galego-Portuguesa de Psicoloxía e Educación, 2I (I), I75-194.

SAK, U. y Ayas, B. (20II) Creative Scientific Ability Test (C-SAT). Manuscrito sin publicar.

SAK, U. y AYAS, M. B. (2013) Creative Scientific Ability Test (C-SAT): A new measure of scientific creativity. Psychological Test and Assessment Modeling, 55 (3), 316-329.

Schawartz, A. T.; Bunce, D. M.; Silberman, R. G.; Stanitski, C. L.; Stratton, W. J. y Zipp, A. P. (1997) Chemistry in Context. Application to society. 2. ${ }^{a}$ edición. New York: McGraw-Hill.

Snyder, A.; Mitchell, J.; Bossomaier, T. y Pallier, G. (2004) The creativity quotient: an objective scoring of ideational fluency. Creativity Research Journal, I6 (4), 4I5-4I9. http://dx.doi.org/I0.I207/si5326934crj1604_4 http://dx.doi.org/I0.1080/I0400410409534552

Sternberg, R. J. y Davidson, J. E. (ig99) Insight. En M. A. Runco y S. R. Pritzker (eds.) Encyclopedia of Creativity (vol. II) (pp. 57-69). New York: Elsevier.

Sternberg, R. J. y Lubart, T. I. (I995/1997) Defying the Crowd: Cultivating Creativity in a Culture of Conformity. New York: Free Press. Trad. castellano: La creatividad en una cultura conformista: un desafío a las masas. Barcelona: Paidós Ibérica.

Sternberg, R. J. y O'Hara, L. (2005) Creatividad e inteligencia. CIC (Cuadernos de Información y Comunicación), IO, II3-I49.

Weiping, H. y Philip, A. (2002) A scientific creativity test for secondary school students. International Journal of Science Education, 24 (4), 389-403. http://dx.doi.org/I0.1080/09500690110098912

Weisberg, R. W. (20I4) Case Studies of Genius: Ordinary Thinking, Extraordinary Outcomes. En D. K. Simonton (ed.) The Wiley Handbook of Genius (pp. 139-165). Chichester, uk: John Wiley \& Sons, Ltd.

http://dx.doi.org/IO.IOO2/978III8367377.ch8

\section{Agradecimientos}

Este trabajo se ha realizado con diferentes ayudas: a) Fundación Séneca-Agencia de Ciencia y Tecnología de la Región de Murcia (Ref.: iı896/PHCS/o9). b) Ministerio de Ciencia y Tecnología (EDU20IO-16370). 